\title{
Generation of liver-specific TGF- $\alpha$ and $c$-Myc-overexpressing fibroblasts for future creation of a liver cancer porcine model
}

\author{
JEEYOUNG KIM*, HUIJEONG AHN*, HEUNG-MYONG WOO, EUNSONG LEE and GEUN-SHIK LEE \\ College of Veterinary Medicine, Kangwon National University, Chuncheon, Gangwon 200-701, Republic of Korea
}

Received October 28, 2013; Accepted March 28, 2014

DOI: $10.3892 / \mathrm{mmr} .2014 .2217$

\begin{abstract}
Liver cancer is one of the most serious life-threatening diseases in the world. Although the rodent model of hepatocellar carcinoma (HCC) is commonly used, it is limited when considering preclinical applications, including transarterial chemoembolization. The pig is a more appropriate model for applying preclinical procedures as it has similar anatomical and physiological characteristics to humans. In the current study, transgenic fibroblasts were generated that overexpressed two proto-oncogenes specifically in hepatocytes. Porcine TGF- $\alpha$ and $c$-myc genes were isolated and these were linked with the porcine albumin promoter, which has exhibited selective activity in liver cells. Targeting vectors were introduced into the porcine fibroblasts using a liposome-mediated delivery system and the transgenic cell line was screened with 3 weeks of G-418 treatment. Selected vector-positive colonies were further confirmed with polymerase chain reaction-based genotyping. Thus, the transgenic cell lines created in the current study should induce liver cancer in pig models following somatic cell nuclear transfer.
\end{abstract}

\section{Introduction}

Liver cancer is one of the most life-threatening diseases in the world, and encompasses several histologically different primary hepatic malignancies, including cholagiocarcinoma, hepatoblastoma and hemangiosarcoma. However, the most common type of liver cancer is hepatocellular carcinoma (HCC) which accounted for 70-85\% of all cases in 2011 (1). Liver carcinogenesis is a multistep process. The presence of specific risk factors promotes gene damage, leading to a cascade of molecular and cellular deregulation that ultimately results in transformation of hepatocytes (2). The ideal therapy for HCC is surgical resec-

Correspondence to: Professor Geun-Shik Lee, College of Veterinary Medicine, Kangwon National University, Chuncheon, Gangwon 200-701, Republic of Korea

E-mail: leegeun@kangwon.ac.kr

*Contributed equally

Key words: liver cancer, pig model, transgenic cell line tion and transplantation, but a lack of liver donors limits the use of these methods. Thus far, transarterial chemoembolization (TACE) is the preferred treatment choice for liver cancer and improves the survival rate of patients (3). Regardless of extensive research into procedures, devices and the application of anti-cancer agents for TACE, the absence of a suitable animal model to replace the rodent model has been the major factor impeding progression in this field.

Several genetically engineered models of HCC have been developed since the early 1980s when the transgenic mouse technique was first introduced (4). Transgenic mice expressing Simian virus 40 large T-antigen in their hepatocytes developed $\mathrm{HCC}$ at the age of 8 months (5). In another study, all transforming growth factor $\alpha$ (TGF- $\alpha$ ) and $c$-Myc (Myc) transgenic male mice presented HCC within 8 months (6). Establishing successful animal models of HCC is crucial for basic and translational studies of HCC. A plethora of HCC mouse models are currently available, which has provided researchers with the opportunities to assess tumor-host interactions, perform drug screenings, mimic the complex multistep process of liver carcinogenesis and conduct various therapeutic experiments (2). However, no mouse model is ideal for the purpose of studying surgical procedures and devices, due to their small body size relative to humans. Thus, alternative models are required to overcome this size limitation.

One of the best candidates is the pig model, as pigs possess anatomical and physiological characteristics similar to those of humans (7). Studies have previously been conducted in pig models of human diseases using transgenic and somatic cell nuclear transfer (SCNT) technologies $(8,9)$. In the present study, the hepatocyte-specific TGF- $\alpha$ and Myc overexpression strategy of previous mouse models $(4,6)$ was adopted in order to allow for the generation of a pig model of HCC in future. To minimize off-target expression of the two proto-oncogenes in the present study, progressive in vitro experiments were performed using a vector constructed in liver and kidney cell lines. The resulting transgenic cell lines were obtained and the insertion of the transgenes in their genomic DNA was checked to confirm that they could be used as donor cells for SCNT.

\section{Materials and methods}

Cell culture. Unless otherwise indicated, all cells were grown at $37^{\circ} \mathrm{C}$ in $5 \% \mathrm{CO}_{2}$, and all cell culture materials were obtained from PAA Laboratories GmbH (Pasching, Austria). 
Table I. Primer details.

\begin{tabular}{|c|c|c|c|c|}
\hline Name & GenBank ID & Direction & $\begin{array}{l}\text { Restriction } \\
\text { enzyme }\end{array}$ & Sequence (5' to $3^{\prime}$ ) \\
\hline \multirow[t]{2}{*}{ Myc cDNA } & NM_001005154 & $\mathrm{F}$ & SalI & TGGACGCTGGATTTCCTTCGGATA \\
\hline & & $\mathrm{R}$ & BamHI & TTATGGGCAAGAGTTCCGTAGCTG \\
\hline \multirow[t]{2}{*}{ TGF- $\alpha$ cDNA } & NM_214251 & $\mathrm{F}$ & EcoRI & CGTAAAATGGTCCCCTCGGCTGGA \\
\hline & & $\mathrm{R}$ & BamHI & TCAGACCACTGTTTCTGAGTGGCA \\
\hline Porcine albumin promoter $(-3,044 \mathrm{nt})$ & NC_010450 & $\mathrm{F}$ & NheI & TCTCTTCTAAATGATCAGCATATA \\
\hline Porcine albumin promoter (-2,033 nt) & & $\mathrm{F}$ & NheI & TTGCAGTGCAAAGTAGGTGGAGAG \\
\hline Porcine albumin promoter (-1,036 nt) & & $\mathrm{F}$ & NheI & TCAGAATTTGGGGTGGGAAAAGTA \\
\hline Porcine albumin promoter (-7 nt) & & $\mathrm{R}$ & HindIII & AAAGGCTTGTGGGGTTGATA \\
\hline \multirow[t]{2}{*}{ Human albumin mRNA } & NM_000477 & F & & ACTTTTATGCCCCGGAACTC \\
\hline & & $\mathrm{R}$ & & TGGAGACTGGCACACTTGAG \\
\hline \multirow[t]{2}{*}{ Human actin mRNA } & NM_001101 & $\mathrm{F}$ & & GGACTTCGAGCAAGAGATGG \\
\hline & & $\mathrm{R}$ & & AGCACTGTGTTGGCGTACAG \\
\hline Confirming primer-1 & NM_001005154 & $\mathrm{F}$ & & GGAAGAGGCGAGAACAGTTG \\
\hline Confirming primer-2 & NM_214251 & F & & TGATACACTGCTGCCAGGTC \\
\hline Confirming primer-3 & IRES & $\mathrm{R}$ & & GAGGAACTGCTTCCTTCACG \\
\hline Confirming primer-4 & NC_010450 & $\mathrm{F}$ & & TGCTTATTCCAGGGGTGTGT \\
\hline Confirming primer-5 & NC_010450 & $\mathrm{R}$ & & AAGCTCCTTCATGTGCAAAA \\
\hline
\end{tabular}

TGF- $\alpha$, transforming growth factor $\alpha$; Myc, $c$-Myc; F, forward; R, reverse; IRES, internal ribosomal entry site - the sequence originated from pIRES2-DsRed-Express ${ }^{\mathrm{TM}}$.

Hep G2 and 293T cells were provided by Dr. Young-Wook Cho (Korean Basic Science Institute, Chuncheon, Korea). To obtain porcine fibroblasts, a pig uterus containing a fetus (male, $\sim 30$ embryonic days old) was transported to the laboratory from a local slaughterhouse (Wonju, Korea). After sterilizing, fetal ears and skin were isolated and minced with a surgical blade in a culture dish (35- or 100-mm; SPL Life Science Co., Gyeonggi-do, Republic of Korea), and subjected to Dulbecco's modified Eagle's medium (DMEM) supplemented with $0.1 \%$ (w/v) trypsin/1 mM EDTA for 1-2 h. Trypsinized cells were subsequently cultured for 6-8 days in DMEM supplemented with $10 \%(\mathrm{v} / \mathrm{v}) \mathrm{FBS}$ and a $10 \mu \mathrm{g} / \mathrm{ml}$ penicillin/streptomycin solution. When the cells were fully confluent, they were collected by trypsinization and were frozen in DMEM supplemented with $40 \%$ FBS and $10 \%$ dimethyl sulfoxide. Porcine peripheral blood monocytes were isolated using lymphocyte separation medium (LSM; Sigma-Aldrich, St. Louis, MO, USA) from freshly drawn peripheral venous blood obtained from domestic pigs. Briefly, EDTA (1.5 mg per $\mathrm{ml}$ of blood; Sigma-Aldrich) treated blood $(10 \mathrm{ml})$ was diluted with equal volumes of PBS and $10 \mathrm{ml}$ of LSM was carefully poured into a centrifuge tube (SPL Life Science Co.). The tube was centrifuged at $500 \times \mathrm{g}$ at room temperature for $30 \mathrm{~min}$ to create a blood-LSM interphase. The mononuclear cell layer was collected into a new tube and diluted with three volumes of PBS. The tube was centrifuged at $500 \mathrm{x} \mathrm{g}$ at room temperature for $10 \mathrm{~min}$. To eliminate RBC contamination, the cell pellet was further treated with RBC lysis buffer (Intron Biotechnology, Seongnam-si, Korea). All cells were cultured in Dulbecco's modified Eagle's medium (DMEM) containing $10 \%$ fetal bovine serum (FBS), $50 \mathrm{U} / \mathrm{ml}$ penicillin and $5 \mu \mathrm{g} / \mathrm{ml}$ streptomycin.
RNA extraction and genomic DNA extraction. Hep G2 cells and peripheral blood mononuclear cells from the pig were subjected to TRIzol reagent (Invitrogen, Carlsbad, CA, USA) to extract total RNA. The RNA concentrations were determined with a spectrophotometer (NanoDrop 2000c; Thermo Scientific, Wilmington, DE, USA) and the RNA was reverse-transcribed into first-strand complementary DNA using Moloney Murine Leukemia Virus Reverse Transcriptase (Invitrogen) according to the manufacturer's instructions. Genomic DNA was isolated by a G-DEX ${ }^{\mathrm{TM}}$ IIc Genomic DNA Extraction kit (Intron Biotechnology).

Polymerase chain reaction (PCR) and quantitative (q)PCR. PCR reactions were performed with LA-Taq polymerase (Takara Bio, Inc., Otsu, Japan) or i-StarTaq polymerase (Intron Biotechnology). The name of PCR fragments, the sequences of primers, the sources of genes (GenBank ID) and the added restriction enzymes are described in Table I. Primers were synthesized by Macrogen (Seoul, Korea). The PCR reactions involved denaturing at $95^{\circ} \mathrm{C}$ for $30 \mathrm{sec}$, annealing at $60^{\circ} \mathrm{C}$ for $30 \mathrm{sec}$, and extension at $72^{\circ} \mathrm{C}$ for $30 \mathrm{sec}$ to $3 \mathrm{~min}$ depending on the size of the products $(1 \mathrm{~min} / \mathrm{kb})$. The PCR products were subjected to cloning processes and/or separated on an agarose gel (1 or 2\%; Invitrogen), stained with ethidium bromide (Invitrogen) and photographed under UV illumination (AE-9150 EZ-Capture II; Atto Corporation, Tokyo, Japan). For gene quantification, qPCR was performed using FG Power SYBR-Green PCR Master mix (Applied Biosystems, Carlsbad, CA, USA) and Eco Real-Time PCR system (Illumina, San Diego, CA, USA).

Vector construction. Unless otherwise indicated, all restriction enzymes and cloning enzymes were obtained from Enzynomics 
A

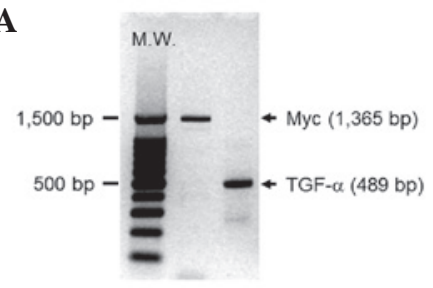

C

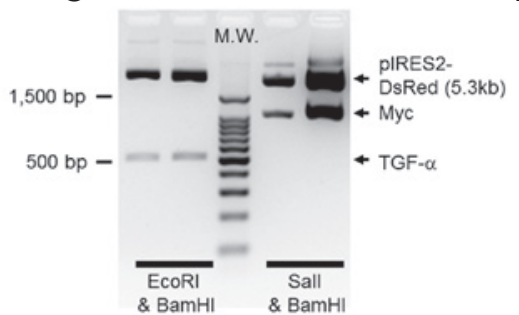

B

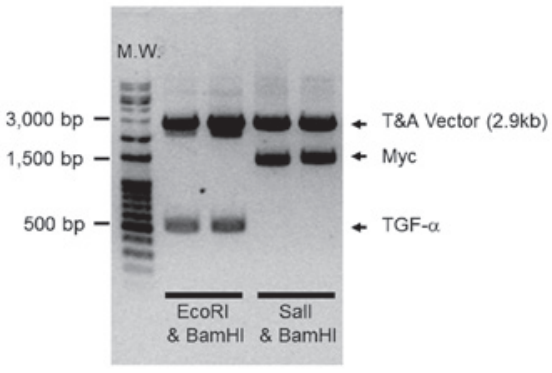

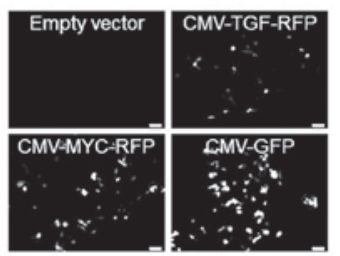

E

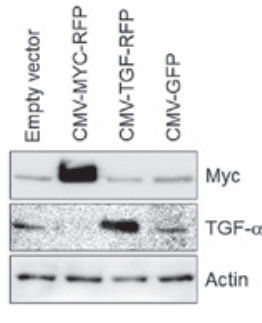

Figure 1. Cloning of porcine TGF- $\alpha$ and Myc genes. (A) Image of agarose gel (1\%) represents the two proto-oncogenes isolated from PBMCs by polymerase chain reaction. (B) Image of TA vector containing TGF- $\alpha$ or Myc cDNAs, captured following digestion with the indicated restriction enzymes. (C) Image represents the confirmation of the expression vectors having incorporated the cDNAs following digestion with the indicated restriction enzymes. (D) The RFP and GFP signals in $293 \mathrm{~T}$ cells after transfection with the indicated plasmids. (E) TGF- $\alpha$ or Myc protein expression was confirmed by immunoblotting following transfection of the indicated plasmids in porcine fetal fibroblasts. M.W., molecular ladder; Myc, $c$-Myc; TGF- $\alpha$, transforming growth factor $\alpha$, PBMC, peripheral blood mononuclear cell; CMV, cytomegalovirus; RFP, red fluorescent protein; GFP, green fluorescent protein.

(Daejeon, Korea). The genes isolated by PCR were cloned into a $\mathrm{yT} \& \mathrm{~A}$ vector (TA vector; RBC Bioscience Corp., Taipei, Taiwan), and the insertion of nucleotide sequences (Macrogen) was confirmed and/or subjected to further sub-cloning. To create the overexpression vector, two proto-oncogenes were inserted into pIRES2-DsRed-Express ${ }^{\mathrm{TM}}$ (Clontech Laboratories, Mountain View, CA, USA). For the luciferase assay, several promoter regions of the porcine albumin (Alb) gene were inserted in pGL4.10 (Promega Corporation, Madison, WI, USA). The selected promoter region was further ligated upstream of the proto-oncogenes of the overexpression vector. The two overexpression vectors, $\mathrm{pAlb}-\mathrm{TGF}-\mathrm{RFP}$ and pAlb-Myc-RFP, were displayed by schematic diagrams using PlasmTM (version 2.1.5.30; http://biofreesoftware.com). Fluorescence signals were observed using a fluorescence microscope (Nikon, Tokyo, Japan).

Transfection and promoter assessment. Transfection was conducted using Lipofectamine ${ }^{\mathrm{TM}} 2000$ (Invitrogen) according to the manufacturer's instructions. 293T cells and Hep G2 cells ( $3 \times 10^{5}$ cells) were plated in 24-well plates (SPL Life Sciences) one day prior to transfection. The promoter constructs $(1.6 \mu \mathrm{g})$ and pRL-TK (3.2 $\mathrm{ng}$; Promega Corporation) were mixed with serum-free DMEM containing Lipofectamine ${ }^{\mathrm{TM}} 2000$ (Invitrogen) and then added to the wells. Following incubation overnight, the cell culture media were replaced with DMEM containing $10 \%$ FBS with $10 \mu \mathrm{g} / \mathrm{ml}$ penicillin/streptomycin solution and incubated for an additional night. Cellular lysates were assayed for luciferase activity using the Dual-Luciferase Reporter Assay system and a GloMax 20/20 Luminometer (Promega Corporation). The relative luciferase activity (\%) was calculated as luciferase activity/Renilla luciferase activity.
Western blotting. The cell lysates were prepared using mild lysis buffer $(150 \mathrm{mM} \mathrm{NaCl}, 1 \%$ Triton X-100, $50 \mathrm{mM}$ Trisbase, $\mathrm{pH}$ 8.0; Sigma-Aldrich). Western blots were prepared, probed at $4^{\circ} \mathrm{C}$ overnight with $c$-Myc antibody (SC-40), TGF- $\alpha$ antibody (SC-36) or actin antibody (SC-1615), and then further probed at room temperature for $3 \mathrm{~h}$ with the horseradish peroxidase-conjugated secondary antibodies (SC-2005 or SC2020; Santa Cruz Biotechnology, Inc., Santa Cruz, CA, USA). The membranes were visualized using PowerOpti-ECL solution (BioNote, Hwasung, Korea) as described in the manufacturer's instructions and images were captured using the EZ-Capture II (Atto Corporation).

Establishment of transgenic cell lines. To determine an appropriate G-418 (Roche, Indianapolis, IN, USA) concentration, 500 porcine fetal fibroblasts were seeded in 96-well plates (SPL Life Sciences) and were treated with various dosages $(15.7,31.1,62.3,125,250,500,1,000$ and $2,000 \mu \mathrm{g} / \mathrm{ml})$ of G-418 or triton X-100 (0.01\%; Sigma-Aldrich) for 3 days. The survival rate was measured using Cell Counting kit- $8^{\mathrm{TM}}$ (Dojindo, Kumamoto, Japan). To create stable cell lines, porcine fetal fibroblasts $\left(5 \times 10^{6}\right.$ cells) were plated in a $100-\mathrm{mm}$ culture dish (SPL Life Sciences) 1 day prior to transfection, and transfected with $24 \mu \mathrm{g}$ linearized overexpression vectors using Lipofectamine ${ }^{\mathrm{TM}} 2000$ (Invitrogen). Following incubation for $6 \mathrm{~h}$, the media were replaced with DMEM containing $10 \% \mathrm{FBS}$ and $125 \mu \mathrm{g} / \mathrm{ml} \mathrm{G}-418$ for $24 \mathrm{~h}$. The concentration of G-418 was gradually reduced to $30 \mu \mathrm{g} / \mathrm{ml}$ at which point colony formation occurred and then the colony was transported to a 6-well plate (SPL Life Sciences) in order to increase the cell number for further study. Fully confluent colonies in the 6-well plate were divided into two portions and subjected to PCR-based genotyping or stored $\left(5 \times 10^{5}\right.$ cells per vial) in the liquid nitrogen tank until required for SCNT in future studies. 

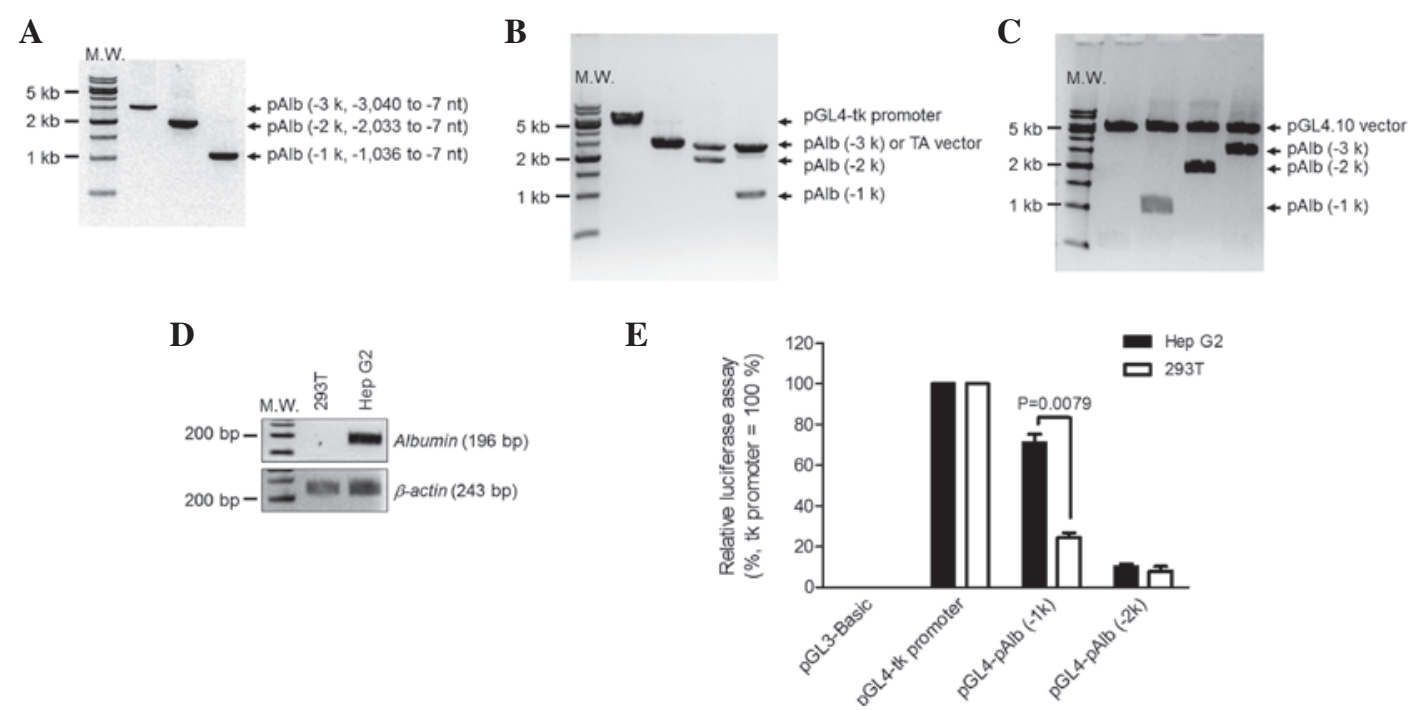

Figure 2. Hepatocyte-specific promoter study. (A) Various regions (+1; transcriptional starting site) of the porcine albumin promoters were isolated by polymerase chain reaction and confirmed using agarose gel electrophoresis. (B) TA vectors containing the promoters were confirmed by restriction enzyme digestion, using NheI and HindIII. (C) The promoters were further cloned into pGL4.10 for relative luciferase assay. (D) The albumin mRNA expression in the liver cell line (Hep G2) or the kidney cell line (293T). (E) Relative luciferase assay measured in liver (Hep G2) and kidney (293T) cells after transient transfection of the vector of panel (C). pRL-TK was co-transfected to measure transfection efficiency. Promoter activity is represented as the percent induction after being normalized to Renilla activity compared with cells transfected with luciferase activity under the control of a tk promoter (pGS4-tk) as a positive control, which was set at $100 \%$. Data represent the mean \pm standard error of the mean of five independent experiments. MW, molecular ladder; Alb, albumin.

Data analysis and ethics. A statistical analysis was performed using GraphPad Prism (version 5 for Windows, GraphPad Software, San Diego, CA, USA). The present study was approved by the ethics committee of Kangwon National University (Chuncheon, Republic of Korea).

\section{Results}

Cloning of porcine TGF- $\alpha$ and Myc genes. To create a mechanism to induce carcinogenesis in the pig liver, two proto-oncogenes (TGF- $\alpha$ and Myc) were isolated from the porcine peripheral blood monocytes by reverse transcription-PCR (Fig. 1A). The isolated genes were cloned into a TA vector (Fig. 1B) and the nucleotide sequences were confirmed by a sequencing analysis (data not shown). TGF- $\alpha$ or Myc genes were further cloned into the overexpression vector containing the gene for red fluorescent protein (RFP; the marker of translation) conjugated by the internal ribosomal entry site (Fig. 1C). The translational activities were further checked in a cell line by transient transfection of these overexpression vectors controlled by a universal (cytomegalovirus; CMV) promoter. The vectors presented RFP signals, which indicated the successful translation of TGF- $\alpha$ or Myc in vivo (Fig. 1D). In addition, the overexpressed TGF- $\alpha$ and Myc proteins in porcine fibroblasts were confirmed by immunoblotting (Fig. 1E). Thus, two proto-oncogenes were isolated from the pig to induce carcinogenesis in future studies.

Assessment of porcine Alb gene as a promoter. To selectively induce the proto-oncogenes in hepatocytes, the liver-specific promoter Alb (pAlb) was assessed. Several regions of the porcine Alb promoter were isolated from the genomic DNA of fetal fibroblasts using PCR (Fig. 2A) and then cloned into the TA vector (Fig. 2B). The isolated promoters were sub-cloned into the vector encoding luciferase to assay the relative promoter activity (Fig. 2C). To confirm a hepatocytespecific Alb promoter, kidney (293T) and liver (Hep G2) cell lines were used. As expected, albumin mRNA was detected in the Hep G2 cells, but not in the 293T cells (Fig. 2D). The luciferase vectors were transiently transfected into the two cell lines and luciferase enzyme activity was measured (Fig. 2E). pAlb (-1k) represented 70\% of promoter activity in Hep G2 cells when compared with a thymidine kinase (tk) promoter, which was used as a positive control. The same promoter in $293 \mathrm{~T}$ cells represented $<20 \%$ of the activity. However, the luciferase activities of pAlb $(-2 \mathrm{k})$ were lower than that of pAlb (-1k) and not different between cell types. The longest promoter regions, pAlb (-3k), presented very weak promoter activities similar to a negative control, pGL3-Basic. On this account, the pAlb (-3k) data were eliminated from Fig. 2E. This indicated that the pAlb $(-1 \mathrm{k})$ was the best candidate to selectively express the two proto-oncogenes in hepatocytes. Thus, the porcine promoter region (-1,036 to $-7 \mathrm{nt})$ was selected to induce hepatocyte-specific expression.

Construction of overexpression vectors to induce HCC in a pig model. For the final transgenic vectors, the Alb promoter was transfected into the vectors that overexpress the two proto-oncogenes (Fig. 3A). Two overexpression vectors are represented in Fig. 3B and these were confirmed by the digestion patterns in Fig. 3C. The functional properties of the vectors were further confirmed with RFP (Fig. 3D) and Myc (Fig. 3E) expression in the kidney and liver cell lines. As expected, the two overexpressing vectors controlled by the CMV presented strong RFP signals and high protein levels of Myc in the cell lines compared with those of the corresponding controls. The Alb promoter controlling the proto-oncogene-expressing vectors induced RFP signals and displayed Myc expression in the Hep G2 cells but not in the 293T cells. This indicated that the transgenic vectors 
A

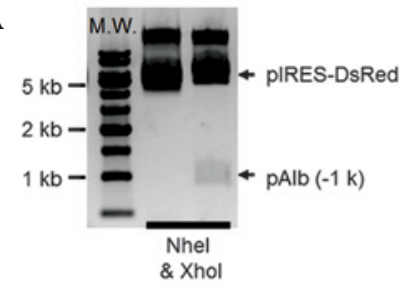

C

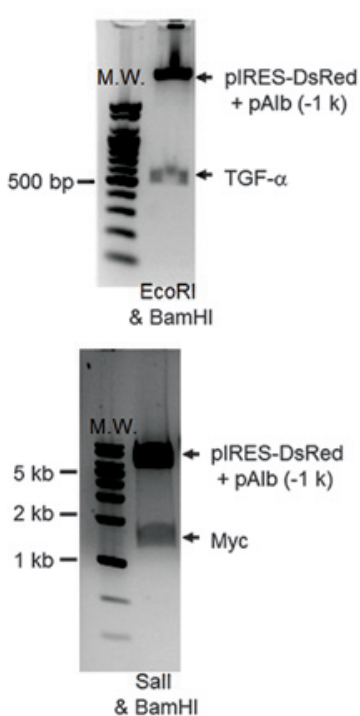

B

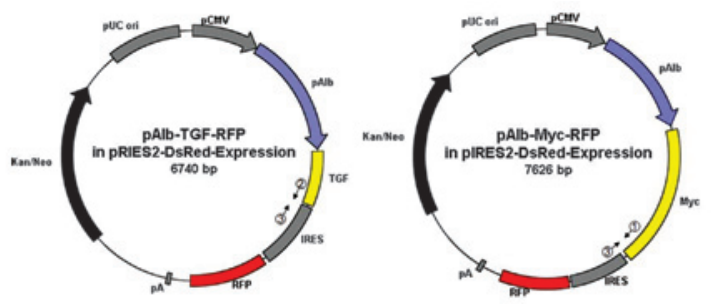

D
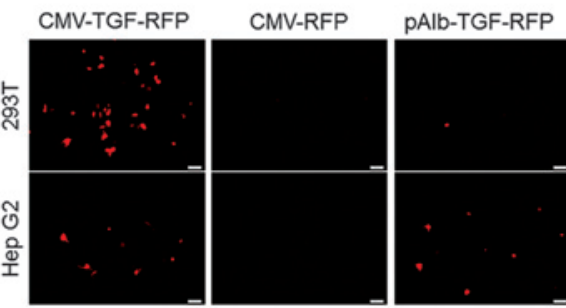

$\mathbf{E}$

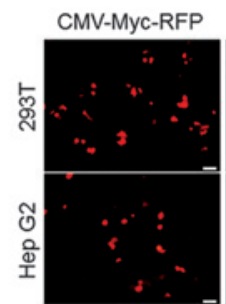

CMV-RFP

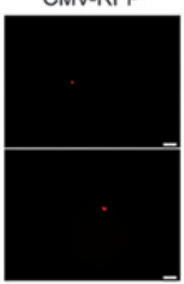

pAlb-Myc-RFP

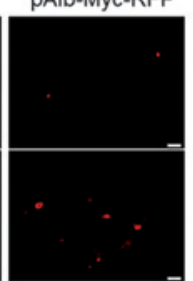

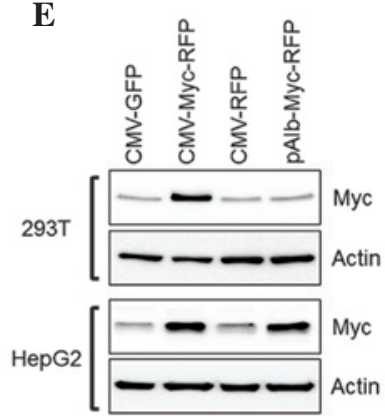

Figure 3. Construction and confirmation of the TGF- $\alpha$ and Myc overexpression vectors. (A) Liver-specific albumin promoter was further cloned into pIRES2-DsRed-Express ${ }^{\mathrm{TM}}$ (pIRES-DsRed) and confirmed by the indicated restriction enzymes. (B) Schematic diagrams displaying the two overexpression vectors (TGF- $\alpha$, left and Myc, right) were drawn by Plasm ${ }^{\mathrm{TM}}$ (version 2.1.5.30; http://biofreesoftware.com). Circled numbers indicate the location of the confirming primers and direction (arrow). (C) The two overexpression vectors were confirmed by the indicated restriction enzymes. (D) RFP expression in liver (Hep G2) and kidney (293T) cells following transient transfection of the indicated plasmids was elucidated under a fluorescence microscope. (E) Myc protein expression was further confirmed with immunoblotting using the lysate from the cells in panel (D). MW, molecular ladder; TGF, transforming growth factor; Myc, $c$-Myc; RFP, red fluorescent protein; CMV, cytomegalovirus.

A

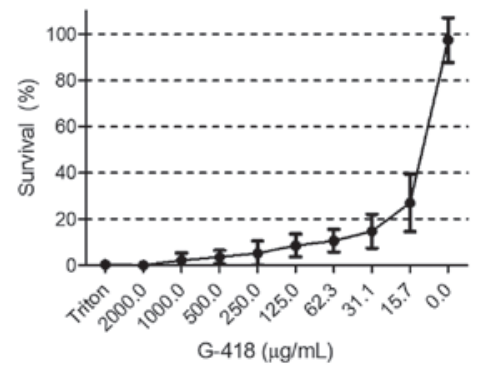

B

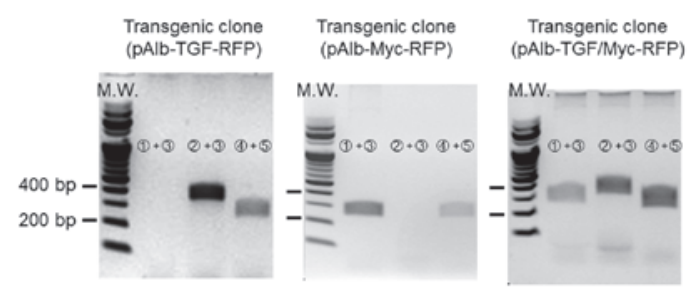

Figure 4. Generation of transgenic cell lines. (A) Cytotoxicity of G-418 was measured after applying the indicated dosage of G-418 to porcine fetal fibroblasts for 3 days. Triton X-100 $(0.01 \%$, Triton) presents complete cell death within $24 \mathrm{~h}$. The Triton-treated group survival rate was $0 \%$ and the non-treated group $(0.0)$ survival rate was $100 \%$. Data represent the mean \pm standard deviation of three independent experiments, each performed in triplicate. (B) The clones positively selected by G-418 were confirmed by polymerase chain reaction-based genotyping. Circled numbers indicate the confirming primers of Table I. $1+3$, 287 bp of Myc overexpression vector; $2+3,353$ bp of TGF- $\alpha$ overexpression vector; 4+5, 274 bp of the exon I region of the endogenous albumin gene. MW, molecular ladder; TGF, transforming growth factor; Myc, $c$-myc; RPF, red fluorescence protein.

had functionally induced two proto-oncogenes specifically in hepatocytes.

Generation of transgenic cell lines to be used as the source of nuclear transfer. To generate transgenic cell lines for SCNT, the transgenic vectors were linearized with a restriction enzyme (NheI) and introduced into porcine fetal fibroblasts via a liposome-mediated DNA delivery system. This delivery system avoided unwanted side-effects, which may originate from the viral mediating system. To aid the screening of a positive clone, the cytotoxicity of neomycin was assayed using the fibroblasts; concentrations of $>30 \mathrm{mg} / \mathrm{ml} \mathrm{G}-418$ effectively eliminated ( $<20 \%$ survival rate) non-transgenic cells within 3 days (Fig. 4A). The antibiotic-resistant clones were screened for $>3$ weeks until colony formation occurred and they were then further confirmed using PCR-based genotyping (Fig. 4B). 
The integration rates of the transgenic vectors into genomic DNA were $>500$ copies when compared with the copy of the endogenous porcine albumin gene (data not shown). Thus, three transgenic cell lines (TGF- $\alpha$, Myc, and a combination of the two) were generated and these can be used in future SCNT to produce liver cancer porcine models.

\section{Discussion}

Liver cancer is a lethal disease and the well-defined risk factors for HCC in humans include cirrhosis, chronic hepatitis $\mathrm{B}$ and $\mathrm{C}$ viral infection, chronic alcohol consumption and afatoxin-B1 intake. Animal models are widely used to improve our understanding of HCC, in particular mouse models (4). These mouse models include the carcinogen-induced model, the implantation model, the genetically engineered mouse, and the viral hepatocarcinogenesis model, which are distinguished by etiological aspects (4). However, none of the currently available mouse models meet all the criteria for the ideal animal model, including biological, genetic, etiological and therapeutic criteria (10).

The most frequently used method for creating a model for HCC is carcinogen treatment, and numerous chemicals have been shown to induce tumors in the mouse liver $(11,12)$. Hepato-carcinogens induce cancer via genotoxic and/or epigenetic (or non-genotoxic) effects. The genotoxic carcinogens induce genetic changes in the target cell, so that it develops into a pre-neoplastic state. The epigenetic carcinogens stimulate the pre-neoplastic state to evolve into a malignant neoplasm by controlling cell proliferation, apoptosis and cell differentiation without DNA modification (12-14). Common hepatocarcinogens include diethylnitrosamine and phenobarbital $(4,15)$. These chemicals are either administered to newborn mice in order to determine genotoxicity, or for longer periods to induce epigenetic carcinogenesis $(11,16)$. Although carcinogen-induced mouse models for HCC are useful for establishing an association between carcinogen exposure and specific genetic changes, the influences of gender, age and the genetic background of the mice on the predictability of HCC development remain disadvantages of these models (17).

More than $80 \%$ of HCCs in humans are attributable to infection with either the hepatitis B virus (HBV), the hepatitis $\mathrm{C}$ virus (HCV) or infection with both (18). This virus-mediated HCC is characteristically preceded by liver cirrhosis and may take more than two decades to develop, implying that hepatocarcinogenesis caused by viral hepatitis requires multiple steps of genetic alterations (19). In addition, $\mathrm{HBV}$ and HCV require the presence of human hepatocytes to induce hepatitis, due to the stringent human tropism of these viruses $(20,21)$. Although viral hepatitis is the main cause of $\mathrm{HCC}$, the relatively long pathogenesis and the lack of the virus inducing $\mathrm{HCC}$ in porcine models restrict us from using this method of viral hepatocarcinogenesis.

Although the exact genetic events in hepatocarcinogenesis are not clear, there is evidence that the $\mathrm{p} 53, \mathrm{Rb}$ and $\mathrm{Wnt} / \beta$-catenin pathways are involved $(22,23)$. Several transgenic mouse lines that are currently used to induce the formation of HCCs are transgenic in one of these pathways (4). Of the transgenic mice expressing the Simian virus 40 large
T-antigen that are directed to the liver by the promoter of antithrombin-III, albumin and $\alpha$-1-antitrypsin, the majority developed hepatocarcinoma within one year $(5,24,25)$. The T-antigen causes malignant transformation of the host cell primarily by inactivating the tumor-suppressor genes; P53 and $\mathrm{Rb}(26,27)$. A double transgenic mouse model overexpressing TGF- $\alpha$ and Myc developed HCC substantially quicker and at a higher rate than the single transgenic 8 -month-old mice $(100 \%$ of males and $30 \%$ of females) (4). This gender-dependent carcinogenesis is similar to the human etiology (4). Based on the mouse models, it is expected that the transgenic cells originating from the pigs in the current study will induce HCC at an early age.

Although surgical approaches such as liver resection and transplantation are considered the most effective treatments to cure HCC, a large portion of patients are unsuitable candidates for these approaches due to the development of multicentric tumors, extrahepatic metastases and early vascular invasion, in addition to a shortage of donor organs, a high complication rate and comorbidities (28-32). Local methods of tumor ablation including TACE, percutaneous ethanol injection, radiofrequency ablation (33-35), microwave coagulation therapy and laser-induced thermotherapy are commonly used (36). TACE has become one of the most common forms of interventional therapy due to its low systemic toxicity and high therapeutic results (37-39). Although several methods and devices for treating HCC have been developed and applied in clinical settings, these are still limited by the lack of an appropriate animal model. To replace the current rodent model, we suggest a pig model, which has similar body size and physiological aspects to humans.

Pigs are one of the major animal species used in translational research and are being used as an alternative to the dog and monkey as the non-rodent of choice in preclinical research (7). Multiple technical procedures for the use of pigs in translational and preclinical studies are available, and numerous studies regarding the anatomy, physiology and pathology of the pig are also available $(40,41)$. Pigs have been the preferred option as a model for surgical training and research into methods including interventional catheter techniques, complex trauma procedures and endoscopic procedures. Pigs are also ideal animals for the development of devices and techniques, and the US Food and Drug Administration has previously accepted data from pigs (7). This supports the rationale for the use of pigs as a liver cancer disease model and an alternative choice of non-rodent species.

In the present study, transgenic cell lines that contained two well-known proto-oncogenes (TGF- $\alpha$ and Myc) controlled by porcine albumin promoter were generated, and they may induce HCC in a porcine model. The expression of a combination of two proto-oncogenes was adopted to maximize carcinogenesis, based on previous mouse models (4). The albumin promoter was the best candidate to selectively express the genes in hepatocytes and it was demonstrated that the selected porcine albumin promoter region was highly active in the liver cell line (Hep G2) but not in the kidney cell line (293T). Although HCC occurrence in the pig model was not demonstrated in the present study, the current transgenic cells have the potential to generate an HCC pig model to replace the classical rodent model, which has multiple limitations. 


\section{Acknowledgements}

The current study was supported by a grant (no. PJ009069) from the Next-Generation BioGreen 21 Program, Rural Development Administration, Korea.

\section{References}

1. Jemal A, Bray F, Center MM, et al: Global cancer statistics. CA Cancer J Clin 61: 69-90, 2011.

2. Li Y, Tang ZY and Hou JX: Hepatocellular carcinoma: insight from animal models. Nat Rev Gastroenterol Hepatol 9: 32-43, 2011.

3. Lencioni R: Chemoembolization for hepatocellular carcinoma Semin Oncol 39: 503-509, 2012.

4. Leenders MW, Nijkamp MW and Borel Rinkes IH: Mouse models in liver cancer research: a review of current literature. World J Gastroenterol 14: 6915-6923, 2008.

5. Dubois N, Bennoun M, Allemand I, et al: Time-course development of differentiated hepatocarcinoma and lung metastasis in transgenic mice. J Hepatol 13: 227-239, 1991

6. Santoni-Rugiu E, Nagy P, Jensen MR, et al: Evolution of neoplastic development in the liver of transgenic mice co-expressing c-myc and transforming growth factor-alpha. Am J Pathol 149: 407-428, 1996.

7. Swindle MM, Makin A, Herron AJ, et al: Swine as models in biomedical research and toxicology testing. Vet Pathol 49: 344-356, 2012.

8. Jung EM, Kim YK, Lee GS, et al: Establishment of inducible cAMP early repressor transgenic fibroblasts in a porcine model of human type 1 diabetes mellitus. Mol Med Rep 6: 239-245, 2012.

9. Kim YK, Lee GS, Jung EM, et al: Generation of fibroblasts overexpressing liver-specific PEPCK in a miniature pig mode of human type 2 diabetes mellitus. Mol Med Rep 6: 45-50, 2012.

10. Hann B and Balmain A: Building 'validated' mouse models of human cancer. Curr Opin Cell Biol 13: 778-784, 2001.

11. Huff J, Cirvello J, Haseman J and Bucher J: Chemicals associated with site-specific neoplasia in 1394 long-term carcinogenesis experiments in laboratory rodents. Environ Health Perspect 93 247-270, 1991

12. Wogan GN: Impacts of chemicals on liver cancer risk. Semin Cancer Biol 10: 201-210, 2000.

13. Gonzalez FJ: The peroxisome proliferator-activated receptor alpha (PPARalpha): role in hepatocarcinogenesis Mol Cell Endocrinol 193: 71-79, 2002.

14. Williams GM: Chemicals with carcinogenic activity in the rodent liver; mechanistic evaluation of human risk. Cancer Lett 117 175-188, 1997.

15. Lee GH: Paradoxical effects of phenobarbital on mouse hepatocarcinogenesis. Toxicol Pathol 28: 215-225, 2000.

16. Chen CJ, Yu MW and Liaw YF: Epidemiological characteristics and risk factors of hepatocellular carcinoma. J Gastroenterol Hepatol 12: S294-308, 1997.

17. Hirst GL and Balmain A: Forty years of cancer modelling in the mouse. Eur J Cancer 40: 1974-1980, 2004.

18. Parkin DM: The global health burden of infection-associated cancers in the year 2002. Int J Cancer 118: 3030-3044, 2006.

19. Fattovich G, Stroffolini T, Zagni I and Donato F: Hepatocellular carcinoma in cirrhosis: incidence and risk factors. Gastroenterology 127 (Suppl 1): S35-S50, 2004.

20. Dandri M, Volz TK, Lütgehetmann M and Petersen J: Animal models for the study of $\mathrm{HBV}$ replication and its variants. J Clin Virol 34 (Suppl 1): S54-S62, 2005.
21. Kremsdorf D and Brezillon N: New animal models for hepatitis $\mathrm{C}$ viral infection and pathogenesis studies. World J Gastroenterol 13: 2427-2435, 2007.

22. Buendia MA: Genetics of hepatocellular carcinoma. Semin Cancer Biol 10: 185-200, 2000.

23. Colnot S, Decaens T, Niwa-Kawakita M, et al: Liver-targeted disruption of Apc in mice activates beta-catenin signaling and leads to hepatocellular carcinomas. Proc Natl Acad Sci USA 101: 17216-17221, 2004.

24. Kitagawa T, Hino O, Lee GH, et al: Multistep hepatocarcinogenesis in transgenic mice harboring SV40 T-antigen gene. Princess Takamatsu Symp 22: 349-360, 1991

25. Sepulveda AR, Finegold MJ, Smith B, et al: Development of a transgenic mouse system for the analysis of stages in liver carcinogenesis using tissue-specific expression of SV40 large T-antigen controlled by regulatory elements of the human alpha-1-antitrypsin gene. Cancer Res 49: 6108-6117, 1989.

26. Ahuja D, Sáenz-Robles MT and Pipas JM: SV40 large T antigen targets multiple cellular pathways to elicit cellular transformation. Oncogene 24: 7729-7745, 2005.

27. Ali SH and DeCaprio JA: Cellular transformation by SV40 large $\mathrm{T}$ antigen: interaction with host proteins. Semin Cancer Biol 11: $15-23,2001$

28. Alsowmely AM and Hodgson HJ: Non-surgical treatment of hepatocellular carcinoma. Aliment Pharmacol Ther 16: 1-15, 2002.

29. Durand F and Belghiti J: Liver transplantation for hepatocellular carcinoma. Hepatogastroenterology 49: 47-52, 2002.

30. Maataoui A, Qian J, Vossoughi D, et al: Transarterial chemoembolization alone and in combination with other therapies: a comparative study in an animal HCC model. Eur Radiol 15: 127-133, 2005.

31. Poon RT, Fan ST, Tsang FH and Wong J: Locoregional therapies for hepatocellular carcinoma: a critical review from the surgeon's perspective. Ann Surg 235: 466-486, 2002.

32. Tang ZY: Treatment of hepatocellular carcinoma. Digestion 59: 556-562, 1998

33. Buscarini E and Buscarini L: Radiofrequency thermal ablation with expandable needle of focal liver malignancies: complication report. Eur Radiol 14: 31-37, 2004.

34. Denys AL, De Baere T, Kuoch V, et al: Radio-frequency tissue ablation of the liver: in vivo and ex vivo experiments with four different systems. Eur Radiol 13: 2346-2352, 2003.

35. Lee JM, Lee YH, Kim YK, et al: Combined treatment of radiofrequency ablation and acetic acid injection: an in vivo feasibility study in rabbit liver. Eur Radiol 14: 1303-1310, 2004

36. Sturm JW, Keese MA, Bönninghoff RG, et al: Locally ablative therapies of hepatocellular carcinoma. Onkologie 24 (Suppl 5): 35-45, 2001 (In German).

37. Achenbach T, Seifert JK, Pitton MB, et al: Chemoembolization for primary liver cancer. Eur J Surg Oncol 28: 37-41, 2002.

38. Llovet JM, Real MI, Montaña X, et al; Barcelona Liver Cancer Group: Arterial embolisation or chemoembolisation versus symptomatic treatment in patients with unresectable hepatocellular carcinoma: a randomised controlled trial. Lancet 359: 1734-1739, 2002

39. Vogl TJ, Trapp M, Schroeder H, et al: Transarterial chemoembolization for hepatocellular carcinoma: volumetric and morphologic CT criteria for assessment of prognosis and therapeutic success - results from a liver transplantation center. Radiology 214: 349-357, 2000.

40. Writing Group Members, Lloyd-Jones D, Adams RJ, Brown TM, et al; American Heart Association Statistics Committee and Stroke Statistics Subcommittee: Heart disease and stroke statistics - 2010 update: a report from the American Heart Association. Circulation 121: e46-e215, 2010.

41. Swindle MM, Smith AC and Hepburn BJ: Swine as models in experimental surgery. J Invest Surg 1: 65-79, 1988. 